\title{
Slepton pair production with aNNLO+NNLL precision
}

\author{
Juri Fiaschi, Michael Klasen and Marthijn Sunder \\ Institut für Theoretische Physik, Westfälische Wilhelms-Universität Münster, \\ Wilhelm-Klemm-Straße 9, D-48149 Münster, Germany \\ E-mail: fiaschi@uni-muenster.de, michael.klasen@uni-muenster.de, \\ mpasunder@uni-muenster.de
}

ABSTRACT: We present a calculation of slepton pair production at the LHC at next-to-nextto-leading logarithmic (NNLL) accuracy, matched to approximate next-to-next-to-leading order (aNNLO) QCD corrections. We collect the relevant analytical formulae, discuss the matching of logarithmically enhanced and fixed-order results and describe the transformation of parton densities and hadronic cross sections to and from Mellin space. Numerically, we find a moderate increase of invariant-mass distributions and total cross sections with respect to our previous results at next-to-leading logarithmic (NLL) accuracy matched to next-to-leading order (NLO), and more importantly a further significant reduction of the factorisation and renormalisation scale dependence that stabilises our predictions to the permil level. The dependence on other supersymmetric parameters like squark and gluino masses and sbottom mixing that enter only at NLO is found to be weak, i.e. less than two percent, as expected.

KEYWORDS: Supersymmetry Phenomenology

ARXIV EPrint: 1911.02419 


\section{Contents}

1 Introduction 1

2 Analytical approach $\quad 2$

2.1 Threshold resummation at NNLL accuracy 3

2.2 Hard matching coefficients up to NNLO 4

2.3 Improvements of the resummation formalism 5

2.4 Fixed-order matching and inverse Mellin transform 6

3 Numerical results for slepton pair production $\quad 7$

3.1 Input parameters 8

3.2 Invariant-mass distributions 8

$\begin{array}{lll}3.3 & \text { Total cross sections } & 10\end{array}$

4 Conclusion $\quad 15$

\section{Introduction}

The search for supersymmetric (SUSY) particles is an important current research topic at CERN's Large Hadron Collider (LHC). The reason is that SUSY is a well-motivated extension of the Standard Model (SM) of particle physics that can solve a significant number of shortcomings of this model. Important examples of SUSY solutions to SM problems are the stabilisation of the Higgs boson mass and a possible candidate for dark matter, which typically is the lightest neutralino, a mixture of the fermionic partners of the neutral electroweak gauge and Higgs bosons [1,2]. Sleptons, the scalar partners of the SM leptons, are usually also among the lightest SUSY particles [3]. While LHC searches already constrain squarks and gluinos, the SUSY partners of quarks and gluons, to the mass range above 1 or $2 \mathrm{TeV}[4,5]$, the limits on left-handed selectron and smuon masses are less stringent and lie at 550 and $560 \mathrm{GeV}$, respectively [6, 7]. Staus can even be as light as $390 \mathrm{GeV}[8,9]$.

Experimental SUSY searches at the LHC rely on precise theoretical predictions that go beyond leading order (LO) in perturbative QCD $[10,11]$ and include not only nextto-leading order (NLO) QCD [12] and SUSY-QCD corrections [13], but that also resum the contributions that are logarithmically enhanced. These enhancements can otherwise spoil the convergence of the perturbative series. They occur at small transverse momenta of the produced slepton pair [14], close to the production threshold [15, 16], or both [17]. Threshold resummation corrections not only increase the production cross section, thereby enhancing the discovery ranges or exclusion limits, but also reduce its dependence on the unphysical factorisation and renormalisation scales and thus render the theoretical 
predictions more accurate. Together with resummation-improved parton density functions (PDFs) [18], also the PDF uncertainty can in principle be reduced [19, 20], even though in practice these PDFs must currently be fitted to smaller data sets than global NLO analyses and thus still have larger errors. Similar calculations have been performed for gaugino and higgsino pairs [21-26], gluinos and gauginos [27-29], and additional gauge bosons [30-33], are available within the public code RESUMMINO [34] and are regularly employed in the experimental analyses by ATLAS [35] and CMS [36]. Predictions have also recently been made for the high-luminosity (HL) and high-energy (HE) phases of the LHC [37].

In this paper, we take our precision calculations for slepton pair production to the next level by resumming not only the leading and next-to-leading logarithms (NLL), but also the next-to-next-to-leading logarithms (NNLL) and matching them not only to the full NLO QCD and SUSY-QCD corrections, but also an approximate next-to-next-toleading order (aNNLO) calculation in QCD. The corresponding analytical formulae are available in the literature [38-40] and are collected here to make the paper self-contained. Similar calculations, based on full NLO SUSY-QCD calculations [41, 42], have also been performed previously for squarks and gluinos [43] and stops [44] and are available through the public code NNLL-fast [45]. Other groups have employed soft-collinear effective theory for sleptons [46], squarks [47] and gluinos [48] and stops [49, 50] with similar conclusions.

The paper is organised as follows: In section 2, we describe our analytical approach and in particular how threshold logarithms can be resummed at NNLL accuracy, matched to a fixed-order calculation up to NNLO and how the PDFs and hadronic cross sections are transformed to and from Mellin space. Our numerical results are contained in section 3. This section starts with a discussion of the QCD and SUSY input parameters, followed by a demonstration of how the NNLL and aNNLO contributions affect the differential cross section in particular at high invariant masses. We then show the effects of the new contributions on the total cross section, its dependence on the factorisation and renormalisation scales as well as on other SUSY parameters like the squark and gluino masses or the trilinear coupling governing squark mixing in the bottom sector. The ensuing conclusions are presented in section 4 .

\section{Analytical approach}

The hadronic invariant mass distribution for the production of slepton pairs,

$$
\begin{aligned}
M^{2} \frac{d \sigma_{A B}}{d M^{2}}(\tau)= & \sum_{a, b} \int_{0}^{1} d x_{a} d x_{b} d z\left[x_{a} f_{a / A}\left(x_{a}, \mu_{F}^{2}\right)\right]\left[x_{b} f_{b / B}\left(x_{b}, \mu_{F}^{2}\right)\right] \\
& \times\left[z \sigma_{a b}\left(z, M^{2}, \mu_{R}^{2}, \mu_{F}^{2}\right)\right] \delta\left(\tau-x_{a} x_{b} z\right),
\end{aligned}
$$

is obtained from a convolution of the parton density functions (PDFs) $f_{a, b / A, B}$, that depend on the longitudinal momentum fractions $x_{a, b}$ of the partons $a, b$ in the external hadrons $A, B$ and the factorisation scale $\mu_{F}$, with the partonic cross section $\sigma_{a b}$, that depends on the squared invariant mass of the produced sleptons $M^{2}$, its ratio $z=M^{2} / s$ (whereas 
$\tau=M^{2} / S$ ) to the partonic (hadronic) center-of-mass energy $s(S)$, and the renormalisation and factorisation scales $\mu_{R}$ and $\mu_{F}$, respectively.

While the leading order (LO) cross section [10, 11] and the virtual next-to-leading order $(\mathrm{NLO})$ corrections are proportional to $\delta(1-z)[12,13]$, the kinematic mismatch in the cancellation of infrared divergences among the virtual and real corrections of order $n$ introduces large logarithmic remainders proportional to

$$
\alpha_{s}^{n}\left(\mu_{R}^{2}\right)\left[\frac{\ln ^{m}(1-z)}{1-z}\right]_{+}, \quad \text { where } \quad m \leq 2 n-1,
$$

which close to threshold $(z \rightarrow 1)$ spoil the convergence of the perturbative series in $\alpha_{s}$ and therefore have to be resummed to all orders [51, 52]. After performing a Mellin transformation,

$$
F(N)=\int_{0}^{1} d y y^{N-1} F(y)
$$

of the PDFs and partonic cross section in eq. (2.1), the hadronic cross section $\sigma_{A B}$ factorises, the singular terms in eq. (2.2) turn into large logarithms of the Mellin variable $N$,

$$
\left[\frac{\ln ^{m}(1-z)}{1-z}\right]_{+} \rightarrow \ln ^{m+1} N+\ldots
$$

and the partonic cross section $\sigma_{a b}$ can be written in the exponentiated form

$$
\sigma_{a b}^{(\text {res. })}\left(N, M^{2}, \mu_{R}^{2}, \mu_{F}^{2}\right)=H_{a b}\left(M^{2}, \mu_{R}^{2}, \mu_{F}^{2}\right) \exp \left[G_{a b}\left(N, M^{2}, \mu_{R}^{2}, \mu_{F}^{2}\right)\right]+\mathcal{O}\left(\frac{1}{N}\right) .
$$

Here, the exponent $G_{a b}$ is universal and contains all the logarithmically enhanced contributions in the Mellin variable $N$, while the hard function $H_{a b}$ is independent of $N$, though process-dependent.

\subsection{Threshold resummation at NNLL accuracy}

Up to next-to-next-to-leading logarithmic (NNLL) accuracy, the exponent $G_{a b}$ can be written as

$$
G_{a b}\left(N, M^{2}, \mu_{R}^{2}, \mu_{F}^{2}\right)=L G_{a b}^{(1)}(\lambda)+G_{a b}^{(2)}\left(\lambda, M^{2}, \mu_{R}^{2}, \mu_{F}^{2}\right)+\alpha_{s} G_{a b}^{(3)}\left(\lambda, M^{2}, \mu_{R}^{2}, \mu_{F}^{2}\right),
$$

where $\lambda=\alpha_{s} b_{0} L$ and $L=\ln \bar{N}=\ln \left(N e^{\gamma_{E}}\right)$. The coefficients of the QCD $\beta$-function are denoted by $b_{n}=\beta_{n} /(2 \pi)^{n+1}$, and the first three coefficients are given by $[53,54]$

$$
\begin{aligned}
& b_{0}=\frac{1}{12 \pi}\left(11 C_{A}-2 n_{f}\right), \\
& b_{1}=\frac{1}{24 \pi^{2}}\left(17 C_{A}^{2}-5 C_{A} n_{f}-3 C_{F} n_{f}\right), \\
& b_{2}=\frac{1}{64 \pi^{3}}\left(\frac{2857}{54} C_{A}^{3}-\frac{1415}{54} C_{A}^{2} n_{f}-\frac{205}{18} C_{A} C_{F} n_{f}+C_{F}^{2} n_{f}+\frac{79}{54} C_{A} n_{f}^{2}+\frac{11}{9} C_{F} n_{f}^{2}\right)
\end{aligned}
$$

with $C_{A}=N_{C}=3, C_{F}=\left(N^{2}-1\right) /\left(2 N_{C}\right)=4 / 3$ and the number of active quark flavours $n_{f}=5$. For Drell-Yan-like processes such as slepton or gaugino pair production initiated 
by quarks and antiquarks only, the coefficients $G_{a b}^{(i)}=g_{a}^{(i)}+g_{b}^{(i)}$ with $a=b=q$ can, e.g., be found up to next-to-leading logarithmic (NLL) accuracy in refs. [15, 22]. At NNLL, one also needs [38]

$$
\begin{aligned}
g_{q}^{(3)}(\lambda)= & \frac{A^{(1)} b_{1}^{2}}{2 \pi b_{0}^{4}} \frac{1}{1-2 \lambda}\left[2 \lambda^{2}+2 \lambda \ln (1-2 \lambda)+\frac{1}{2} \ln ^{2}(1-2 \lambda)\right] \\
& +\frac{A^{(1)} b_{2}}{2 \pi b_{0}^{3}}\left[2 \lambda+\ln (1-2 \lambda)+\frac{2 \lambda^{2}}{1-2 \lambda}\right]+\frac{2 A^{(1)}}{\pi} \zeta_{2} \frac{\lambda}{1-2 \lambda} \\
& -\frac{A^{(2)} b_{1}}{(2 \pi)^{2} b_{0}^{3}} \frac{1}{1-2 \lambda}\left[2 \lambda^{2}+2 \lambda+\ln (1-2 \lambda)\right]+\frac{A^{(3)}}{\pi^{3} b_{0}^{2}} \frac{\lambda^{2}}{1-2 \lambda}-\frac{D^{(2)}}{2 \pi^{2} b_{0}} \frac{\lambda}{1-2 \lambda} \\
& +\frac{A^{(1)} b_{1}}{2 \pi b_{0}^{2}} \frac{1}{1-2 \lambda}[2 \lambda+\ln (1-2 \lambda)] \ln \left(\frac{M^{2}}{\mu_{R}^{2}}\right)+\frac{A^{(1)}}{2 \pi}\left[\frac{\lambda}{1-2 \lambda} \ln ^{2}\left(\frac{M^{2}}{\mu_{R}^{2}}\right)-\lambda \ln ^{2}\left(\frac{\mu_{F}^{2}}{\mu_{R}^{2}}\right)\right] \\
& -\frac{A^{(2)}}{2 \pi^{2} b_{0}}\left[\frac{\lambda}{1-2 \lambda} \ln \left(\frac{M^{2}}{\mu_{R}^{2}}\right)-\lambda \ln \left(\frac{\mu_{F}^{2}}{\mu_{R}^{2}}\right)\right] .
\end{aligned}
$$

Here, the universal process-independent coefficients are given by [55]

$$
\begin{aligned}
A^{(1)}=2 C_{F}, & \\
A^{(2)}=2 C_{F} & {\left[C_{A}\left(\frac{67}{18}-\zeta_{2}\right)-\frac{5}{9} n_{f}\right], } \\
A^{(3)}=\frac{1}{2} C_{F}[ & C_{A}^{2}\left(\frac{245}{24}-\frac{67}{9} \zeta_{2}+\frac{11}{6} \zeta_{3}+\frac{11}{5} \zeta_{2}^{2}\right)+C_{F} n_{f}\left(2 \zeta_{3}-\frac{55}{24}\right) \\
& \left.+C_{A} n_{f}\left(\frac{10}{9} \zeta_{2}-\frac{7}{3} \zeta_{3}-\frac{209}{108}\right)-\frac{n_{f}^{2}}{27}\right]
\end{aligned}
$$

and [38]

$$
D^{(2)}=2 C_{F}\left[C_{A}\left(-\frac{101}{27}+\frac{11}{3} \zeta_{2}+\frac{7}{2} \zeta_{3}\right)+n_{f}\left(\frac{14}{27}-\frac{2}{3} \zeta_{2}\right)\right] .
$$

\subsection{Hard matching coefficients up to NNLO}

The hard $N$-independent part of the Mellin-transformed partonic cross section in eq. (2.5),

$$
H_{a b}\left(M^{2}, \mu_{R}^{2}, \mu_{F}^{2}\right)=\sigma_{a b}^{(0)} \mathcal{C}_{a b}\left(M^{2}, \mu_{R}^{2}, \mu_{F}^{2}\right),
$$

can be perturbatively expanded in terms of the Mellin-transformed LO cross section $\sigma_{a b}^{(0)}$ and

$$
\mathcal{C}_{a b}\left(M^{2}, \mu_{R}^{2}, \mu_{F}^{2}\right)=\sum_{n=0}\left(\frac{\alpha_{s}}{2 \pi}\right)^{n} \mathcal{C}_{a b}^{(n)}\left(M^{2}, \mu_{R}^{2}, \mu_{F}^{2}\right)
$$

where the hard matching coefficients

$$
\mathcal{C}_{a b}^{(n)}\left(M^{2}, \mu_{R}^{2}, \mu_{F}^{2}\right)=\left(\frac{2 \pi}{\alpha_{s}}\right)^{n}\left[\frac{\sigma_{a b}^{(n)}}{\sigma_{a b}^{(0)}}\right]_{\mathrm{N}-\text { ind }}
$$

are obtained from the finite ( $N$-independent) terms in the ratio of the $n$-th order cross section over the LO one. The coefficients up to next-to-next-to-leading order (NNLO) can 
be obtained from refs. [39, 40] and are given by

$$
\begin{aligned}
\mathcal{C}_{q \bar{q}}^{(0)}=1, & \\
\mathcal{C}_{q \bar{q}}^{(1)}=C_{F}[ & \left.\frac{4}{3}\left(\pi^{2}-6\right)-3 \log \left(\frac{\mu_{F}^{2}}{M^{2}}\right)\right] \\
\mathcal{C}_{q \bar{q}}^{(2)}=\frac{C_{F}}{720}\{ & 5\left(-4605 C_{A}+4599 C_{F}+762 n_{f}\right)+20 \pi^{2}\left(188 C_{A}-297 C_{F}-32 n_{f}\right) \\
& -92 \pi^{4}\left(C_{A}-6 C_{F}\right)+180\left(11 C_{A}+18 C_{F}-2 n_{f}\right) \log ^{2}\left(\frac{\mu_{F}^{2}}{M^{2}}\right) \\
& -160\left(11 C_{A}-2 n_{f}\right)\left(6-\pi^{2}\right) \log \left(\frac{\mu_{R}^{2}}{M^{2}}\right)+80\left(151 C_{A}-135 C_{F}+2 n_{f}\right) \zeta_{3} \\
& +20 \log \left(\frac{\mu_{F}^{2}}{M^{2}}\right)\left[-51 C_{A}+837 C_{F}+6 n_{f}-4 \pi^{2}\left(11 C_{A}+27 C_{F}-2 n_{f}\right)\right. \\
& \left.\left.+\left(-198 C_{A}+36 n_{f}\right) \log \left(\frac{\mu_{R}^{2}}{M^{2}}\right)+216\left(C_{A}-2 C_{F}\right) \zeta_{3}\right]\right\} .
\end{aligned}
$$

By including the coefficients up to NNLO, the resummation of logarithmically enhanced contributions is improved, since also beyond NNLO in $\alpha_{s}$ the finite terms are multiplied by threshold logarithms.

\subsection{Improvements of the resummation formalism}

While in the limit of large $N$ the cross section is clearly dominated by terms of $\mathcal{O}\left(\ln ^{2} N\right)$, $\mathcal{O}(\ln N)$ and $\mathcal{O}(1)$, some of the terms suppressed by powers of $1 / N$ are multiplied by powers of $\ln N$ and can thus also have a non-negligible effect. In refs. [56-58] the authors showed that these contributions are due to collinear parton emission and can be consistently included in the resummation formula, leading to a collinear-improved resummation formalism. This has recently also been demonstrated using Soft-Collinear Effective Field Theory (SCET) for Drell-Yan [59, 60] and Higgs production [61] as well as with diagrammatic methods for general color-singlet final states [62]. The modification simply amounts to the introduction of the $N$-dependent terms

$$
\begin{aligned}
\mathcal{C}_{q \bar{q}}^{(1)} \rightarrow \widetilde{\mathcal{C}}_{q \bar{q}}^{(1)} & =\mathcal{C}_{q \bar{q}}^{(1)}+A_{1} \frac{\ln \bar{N}-\frac{1}{2} \ln \frac{M^{2}}{\mu_{f}^{2}}}{N}, \\
\mathcal{C}_{q g}^{(1)} \rightarrow \widetilde{\mathcal{C}}_{q g}^{(1)} & =-T_{R} \frac{\ln \bar{N}-\frac{1}{2} \ln \frac{M^{2}}{\mu_{f}^{2}}}{N}
\end{aligned}
$$

in the $C_{a b}^{(n)}$ coefficients of eq. (2.17). As discussed previously, this collinear improvement is implemented in RESUMMINO for both slepton [15] and gaugino [22] pair production.

Furthermore, the exponentiation of the contributions embodied in the $\mathcal{C}$-function has been proved in [63]. As the authors of ref. [63] recognize, this exponentiation of the $N$ independent terms is not comparable to the standard threshold resummation in terms of predictive power. While in the latter case a low-order calculation can be used to predict the behaviour of full towers of logarithms, in the former case it is not possible to directly get 
information on the behaviour of constant terms at, say, $n$ loops, but a complete calculation at the $n^{\text {th }}$ perturbative order will still be necessary [64]. Nonetheless, the comparison of the numerical results obtained with and without the exponentiation of the constant terms can at least provide an estimate of the errors due to missing higher-order corrections. This has very recently been done for SM lepton pair production in figure 5 of ref. [65] with the result that the differences remain below $1 \%$.

\subsection{Fixed-order matching and inverse Mellin transform}

Although near to threshold the resummed cross section is a valid approximation, outside this region the normal perturbative calculation should be used. A reliable prediction in all kinematic regions is then obtained through a consistent matching of the two results with

$$
\sigma_{a b}=\sigma_{a b}^{(\text {res. })}+\sigma_{a b}^{(\text {f.o. })}-\sigma_{a b}^{(\text {exp. })} .
$$

Here, the resummed cross section $\sigma_{a b}^{(\text {res.) }}$ in eq. (2.5) has been re-expanded to NNLO, yielding $\sigma_{a b}^{\text {(exp.) }}$, and subtracted from the fixed-order calculation $\sigma_{a b}^{(\text {f.o. })}$ in order to avoid the double counting of the logarithmically enhanced contributions. At $\mathcal{O}\left(\alpha_{s}^{2}\right)$ we then obtain

$$
\begin{aligned}
\sigma_{a b}^{(\exp .)}(N & \left., M^{2}, \mu_{R}^{2}, \mu_{F}^{2}\right)=\sigma_{a b}^{(0)} \mathcal{C}_{a b}\left(M^{2}, \mu_{R}^{2}, \mu_{F}^{2}\right) \exp \left[G_{a b}\left(N, M^{2}, \mu_{R}^{2}, \mu_{F}^{2}\right)\right] \\
& =\sigma_{a b}^{(0)}\left[1+\left(\frac{\alpha_{s}}{2 \pi}\right) \mathcal{C}_{a b}^{(1)}+\left(\frac{\alpha_{s}}{2 \pi}\right)^{2} \mathcal{C}_{a b}^{(2)}+\ldots\right]\left[1+\left(\frac{\alpha_{s}}{2 \pi}\right) \mathcal{K}^{(1)}+\left(\frac{\alpha_{s}}{2 \pi}\right)^{2} \mathcal{K}^{(2)}+\ldots\right] \\
& =\sigma_{a b}^{(0)}\left[1+\left(\frac{\alpha_{s}}{2 \pi}\right)\left(\mathcal{C}_{a b}^{(1)}+\mathcal{K}^{(1)}\right)+\left(\frac{\alpha_{s}}{2 \pi}\right)^{2}\left(\mathcal{C}_{a b}^{(2)}+\mathcal{K}^{(2)}+\mathcal{C}_{a b}^{(1)} \mathcal{K}^{(1)}\right)+\ldots\right] .
\end{aligned}
$$

The coefficients of the expanded exponential term can be organised in powers of $L$ as

$$
\begin{aligned}
& \mathcal{K}^{(1)}=\mathcal{K}^{(1,1)} L+\mathcal{K}^{(1,2)} L^{2}, \\
& \mathcal{K}^{(2)}=\mathcal{K}^{(2,1)} L+\mathcal{K}^{(2,2)} L^{2}+\mathcal{K}^{(2,3)} L^{3}+\mathcal{K}^{(2,4)} L^{4} .
\end{aligned}
$$

Explicitly, they are given by [39, 40]

$$
\begin{aligned}
& \mathcal{K}^{(1,1)}=4 C_{F} \log \left(\frac{\mu_{F}^{2}}{s}\right), \\
& \mathcal{K}^{(1,2)}=4 C_{F}, \\
& \mathcal{K}^{(2,1)}=-\frac{C_{F}}{27}\left\{56 n_{f}-404 C_{A}+3 \log \left(\frac{\mu_{F}^{2}}{s}\right)\left[20 n_{f}+2 C_{A}\left(-67+3 \pi^{2}\right)\right.\right. \\
& \left.\left.+3\left(11 C_{A}-2 n_{f}\right)\left(\log \left(\frac{\mu_{F}^{2}}{\mu_{R}^{2}}\right)-\log \left(\frac{\mu_{R}^{2}}{s}\right)\right)\right]+378 C_{A} \zeta_{3}\right\}, \\
& \mathcal{K}^{(2,2)}=\frac{2}{9} C_{F}\left[-10 n_{f}+67 C_{A}-3 C_{A} \pi^{2}+36 C_{F} \log ^{2}\left(\frac{\mu_{F}^{2}}{s}\right)\right. \\
& \left.+\left(33 C_{A}-6 n_{f}\right) \log \left(\frac{\mu_{R}^{2}}{s}\right)\right], \\
& \mathcal{K}^{(2,3)}=\frac{4}{9} C_{F}\left[11 C_{A}-2 n_{f}+36 C_{F} \log \left(\frac{\mu_{F}^{2}}{s}\right)\right], \\
& \mathcal{K}^{(2,4)}=8 C_{F}^{2} \text {. }
\end{aligned}
$$


The SUSY-QCD (squark-gluino loop) corrections are only matched at NLO, since they are not known beyond this order [13]. In this sense, our results are accurate to approximate NNLO (aNNLO) plus NNLL precision. This approximation is justified by the fact that the SUSY-QCD corrections are subdominant due to the large squark and gluino masses.

Having computed the resummed and the perturbatively expanded results in Mellin space, we must multiply them with the $N$-moments of the PDFs and perform an inverse Mellin transform,

$$
M^{2} \frac{d \sigma_{A B}}{d M^{2}}(\tau)=\frac{1}{2 \pi i} \int_{\mathcal{C}_{N}} d N \tau^{-N} M^{2} \frac{d \sigma_{A B}(N)}{d M^{2}}
$$

in order to obtain the hadronic cross section as a function of $\tau=M^{2} / S$. Special attention must be paid to the singularities in the resummed exponents $G_{a b}^{(1,2,3)}$, which are situated at $\lambda=1 / 2$ and are related to the Landau pole of the perturbative coupling $\alpha_{s}$. In order to avoid this pole as well as those in the Mellin moments of the PDFs related to the small- $x$ (Regge) singularity $f_{a / A}\left(x, \mu_{0}^{2}\right) \propto x^{\alpha}(1-x)^{\beta}$ with $\alpha<0$, we choose an integration contour $\mathcal{C}_{N}$ according to the principal value procedure proposed in ref. [66] and the minimal prescription proposed in ref. [67]. We define two branches,

$$
\mathcal{C}_{N}: \quad N=C+z e^{ \pm i \phi} \text { with } z \in[0, \infty[
$$

where the constant $C$ is chosen such that the singularities of the $N$-moments of the PDFs lie to the left and the Landau pole to the right of the integration contour. Formally, the angle $\phi$ can be chosen in the range $[\pi / 2, \pi$, but the integral converges faster if $\phi>\pi / 2$. The Mellin moments of the PDFs are obtained by fitting to the parameterisations tabulated in $x$-space the functional form used by the MSTW collaboration [68]

$$
f(x)=A_{0} x^{A_{1}}(1-x)^{A_{2}}\left(1+A_{3} \sqrt{x}+A_{4} x+A_{5} x^{\frac{3}{2}}\right)+A_{6} x^{2}+A_{7} x^{\frac{5}{2}},
$$

which has the advantage that it can be transformed analytically with the result

$$
\begin{aligned}
F(x)= & A_{0} \Gamma(y) \mathrm{B}^{\prime}\left(A_{1}+N, y\right)+A_{3} \mathrm{~B}^{\prime}\left(A_{1}+N+\frac{1}{2}, y\right)+A_{4} \mathrm{~B}^{\prime}\left(A_{1}+N+1, y\right) \\
& +A_{5} \mathrm{~B}^{\prime}\left(A_{1}+N+\frac{3}{2}, y\right)+A_{6} \mathrm{~B}^{\prime}\left(A_{1}+N+2, y\right)+A_{7} \mathrm{~B}^{\prime}\left(A_{1}+N+\frac{5}{2}, y\right) .
\end{aligned}
$$

Here, $y=A_{2}+1$ and $\mathrm{B}^{\prime}(x, y)=\mathrm{B}(x, y) / \Gamma(y)=\Gamma(x) / \Gamma(x+y)$. We verified that we obtain good fits not only for the MMHT2014NLO118 [68], but also for the CT14NLO fits [69] up to large values of $x$ and for all typical factorisation scales, even though the latter are obtained with an ansatz that includes an exponential function.

\section{Numerical results for slepton pair production}

In this section, we present numerical results for slepton pair production at the LHC with aNNLO+NNLL precision. We first discuss our choice of input parameters and demonstrate the impact of threshold resummation on the invariant-mass distributions, after which we show and discuss our experimentally more relevant predictions for the total cross sections as a function of the slepton mass and other, subdominant SUSY parameters. 


\subsection{Input parameters}

Our numerical results for proton-proton collisions at LHC Run 2 with a center-of-mass energy $\sqrt{S}$ of $13 \mathrm{TeV}$ have been obtained with CT14 PDFs [69], which we employ consistently at LO and NLO with the corresponding partonic cross sections. While the PDF uncertainty in resummation calculations can in principle be reduced by using also resummationimproved PDFs [19, 20], the latter are fitted to a substantially smaller data set than those at NLO, which unfortunately currently still results in a larger PDF uncertainty [18]. We therefore use here NLO PDFs with NLL and NNLL partonic cross sections and refer to refs. $[19,20]$ for a detailed discussion of PDF uncertainties. Since top (s)quarks do not enter our calculations, all other five quark flavours are treated as massless, and the QCD scale parameter $\Lambda$ is fixed accordingly to its CT14 values. For our central predictions, the renormalisation and factorisation scales are identified with the slepton mass. For scale uncertainty estimates, we employ the seven-point method, i.e. the scales are varied individually by relative factors of two, but not four.

Based on an integrated LHC luminosity of $139(35.9) \mathrm{fb}^{-1}$ and for sufficiently large mass differences with the lightest neutralino, the ATLAS (CMS) collaboration has recently excluded left-handed selectrons below 550 (400) GeV. For two (not three, as stated in the ATLAS abstract and conclusion) generations of mass-degenerate sleptons, the limit increases to $700(450) \mathrm{GeV}[6,7]$. We therefore adopt for the invariant-mass distributions a default slepton mass of $1 \mathrm{TeV}$ and use $700 \mathrm{GeV}$ as the lower mass limit for the total cross sections. Squarks and gluinos enter only at NLO in virtual loop diagrams, and therefore their masses play only a subdominant role. We adopt a squark and gluino mass of $1.3 \mathrm{TeV}$ as our default value, which is still allowed for not too large mass differences with the lightest neutralino, even though the most stringent ATLAS (CMS) mass limits already reach $1.94(1.63)$ and $2.35(2.31) \mathrm{TeV}$, respectively [4, 5]. We will study the dependence on these parameters up to $2.5 \mathrm{TeV}$ and see that the dependence is indeed weak, as is the dependence on the mixing angle in the case of bottom squarks.

\subsection{Invariant-mass distributions}

In figure 1 (top) we plot the invariant-mass distributions for slepton pair production at the LHC with a center-of-mass energy of $\sqrt{S}=13 \mathrm{TeV}$ and with LO (yellow), NLO (green), $\mathrm{NLO}+\mathrm{NLL}$ (blue) and aNNLO+NNLL (red) precision together with the corresponding scale uncertainties (shaded bands). Since we do not take into account decays or detector acceptances, these results are valid for both left-handed selectrons and smuons of $1 \mathrm{TeV}$ mass, while the cross sections for maximally mixed staus or right-handed selectrons and smuons are typically smaller by about a factor of 2 to 2.5 [19]. The cross section rises with the third power of the slepton velocity and peaks at an invariant mass that is considerably above the minimal value $2 m_{\tilde{\ell}}$ before falling steeply off due to the $s$-channel propagator and the parton luminosity [15].

The effect of the higher-order corrections is best seen in figure 1 (bottom) as ratios ( $K$ factors) of NLO+NLL over NLO (green), aNNLO+NNLL over NLO+NLL (blue) and aNNLO+NNLL over NLO (red) differential cross sections. Resummation effects at NLL 


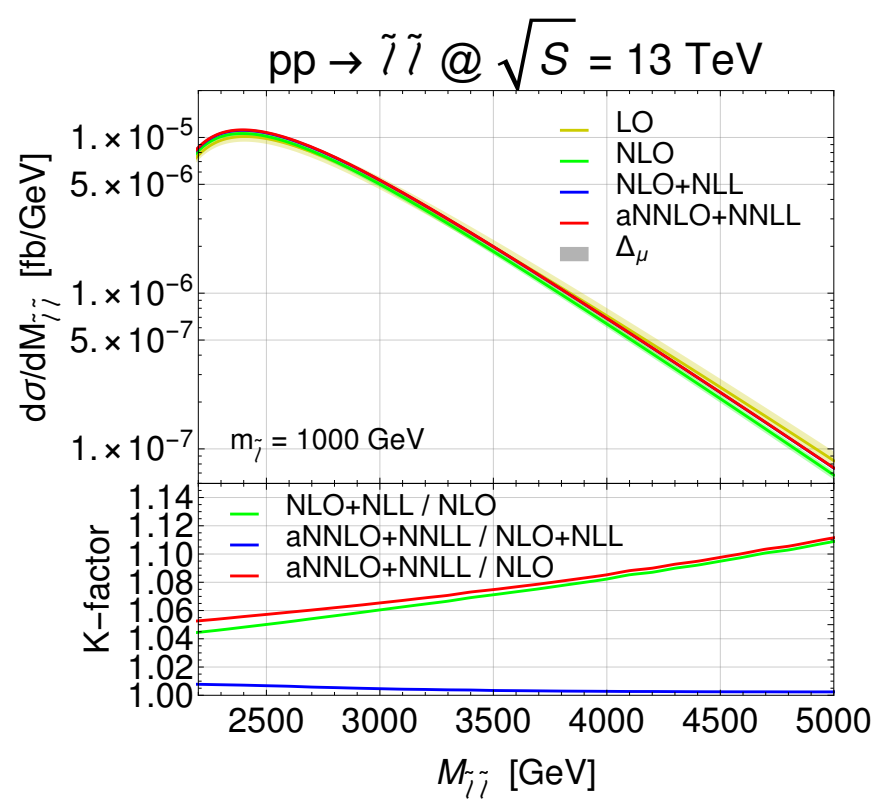

Figure 1. Top: Invariant-mass distribution for left-handed selectron (or smuon) pair production at the LHC with a center-of-mass energy of $\sqrt{S}=13 \mathrm{TeV}$ for a fixed slepton mass of $1 \mathrm{TeV}$. Shown are results at LO (yellow), NLO (green), NLO+NLL (blue) and aNNLO+NNLL (red) together with the corresponding scale uncertainties (shaded bands). Bottom: Ratios ( $K$ factors) of NLO+NLL over NLO (green), aNNLO+NNLL over NLO+NLL (blue) and aNNLO+NNLL over NLO (red) differential cross sections as a function of the invariant mass of the slepton pair.

(green) accuracy become more important with respect to the fixed (NLO) order as the invariant mass of the slepton pair approaches the production threshold. The corresponding $K$ factor increases in the invariant mass range of 2.2 to $5 \mathrm{TeV}$ from 1.045 to 1.110 . The increase from NLO+NLL to aNNLO+NNLL is much smaller as expected for a converging expansion, and most visible at low invariant masses, where the constant terms at aNNLO induce an increase by about $1 \%$. Note that the size of the $K$ factor depends not only on the invariant mass, but also the choice of scales, whose variations must also be considered.

Apart from the increase in cross section, which is small for sleptons, a second important effect of resummation calculations is the reduction in the theoretical uncertainty. It is traditionally estimated by varying the renormalisation and factorisation scales following the seven-point method. The result for the invariant mass distribution is shown in figure 2 . While the uncertainty remained already mostly below one percent and exceeded this value very close to threshold at NLO+NLL (blue), the new contributions at aNNLO+NNLL (red shaded band) reduce the uncertainty considerably further to about one permil. Only at low invariant mass, i.e. far from threshold, the uncertainty rises to about two permil. This demonstrates the excellent stability of the expansion. Remember, however, that there are of course also additional uncertainties from varying the renormalisation [70, 71], factorisation [72, 73] and resummation schemes [56]. 


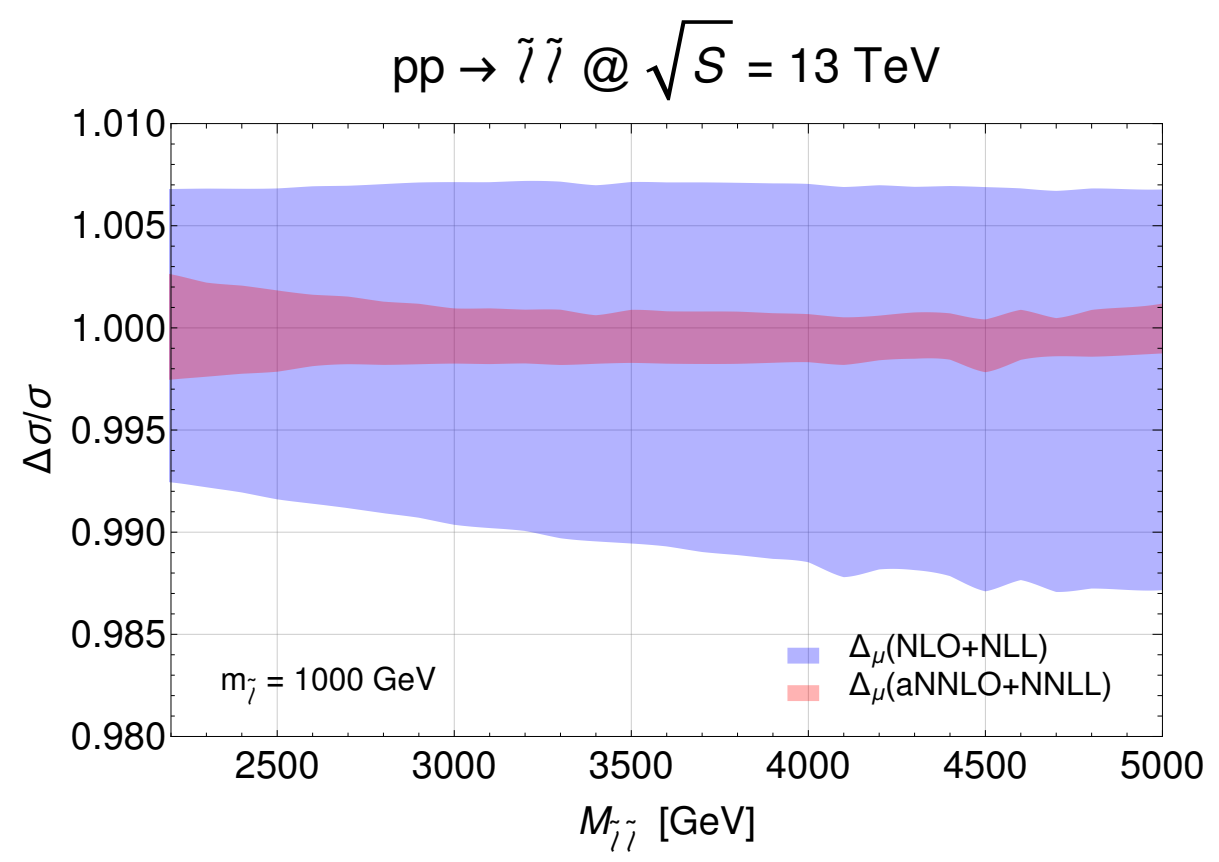

Figure 2. Scale uncertainty of the invariant-mass distribution for left-handed selectron (or smuon) pair production at the LHC with a center-of-mass energy of $\sqrt{S}=13 \mathrm{TeV}$ for a fixed slepton mass of $1 \mathrm{TeV}$. Shown are the results at NLO+NLL (blue) and aNNLO+NNLL (red shaded band).

\subsection{Total cross sections}

We now turn to our predictions for total cross sections for slepton pair production at the LHC, which are directly applicable to determine experimental discovery ranges or exclusion limits. To this end, we plot in figure 3 (top) the total production cross section for lefthanded selectron (or smuon) pairs at the LHC with a center-of-mass energy of $\sqrt{S}=13 \mathrm{TeV}$ as a function of the slepton mass in the range $700 \mathrm{GeV}$ to $1500 \mathrm{GeV}$. In this range, the cross section falls from almost $0.1 \mathrm{fb}$ to below $1 \mathrm{ab}$, corresponding to more than 10 events at $700 \mathrm{GeV}$ with the currently analysed integrated luminosity of $139 \mathrm{fb}^{-1}$ to 3 events at $1 \mathrm{TeV}$ with the LHC Run 3 goal of $300 \mathrm{fb}^{-1}$ and a few events at $1.5 \mathrm{TeV}$ with the highluminosity (HL) LHC goal of $3 \mathrm{ab}^{-1}$. The reduction of the scale uncertainty is visible as a decrease in width of the predictions from LO (yellow shaded band) to the higher orders (other colours).

The $K$ factors in figure 3 (bottom) show that the logarithmic terms at NLL (green) and NNLL (red) first reduce, then enhance the cross section by a few percent with respect to the NLO prediction as the slepton mass increases. The aNNLO $(+\mathrm{NNLL})$ terms lead in addition to an almost constant increase over the $\mathrm{NLO}(+\mathrm{NLL})$ prediction of about one percent (blue).

As for the invariant mass distribution, it is important to study the scale dependence at different levels of precision also for the total cross section. The variation of the total slepton pair production cross section at the LHC with $13 \mathrm{TeV}$ center-of-mass energy with the factorisation scale is shown in figure 4 (top), normalised to the cross section at the central 


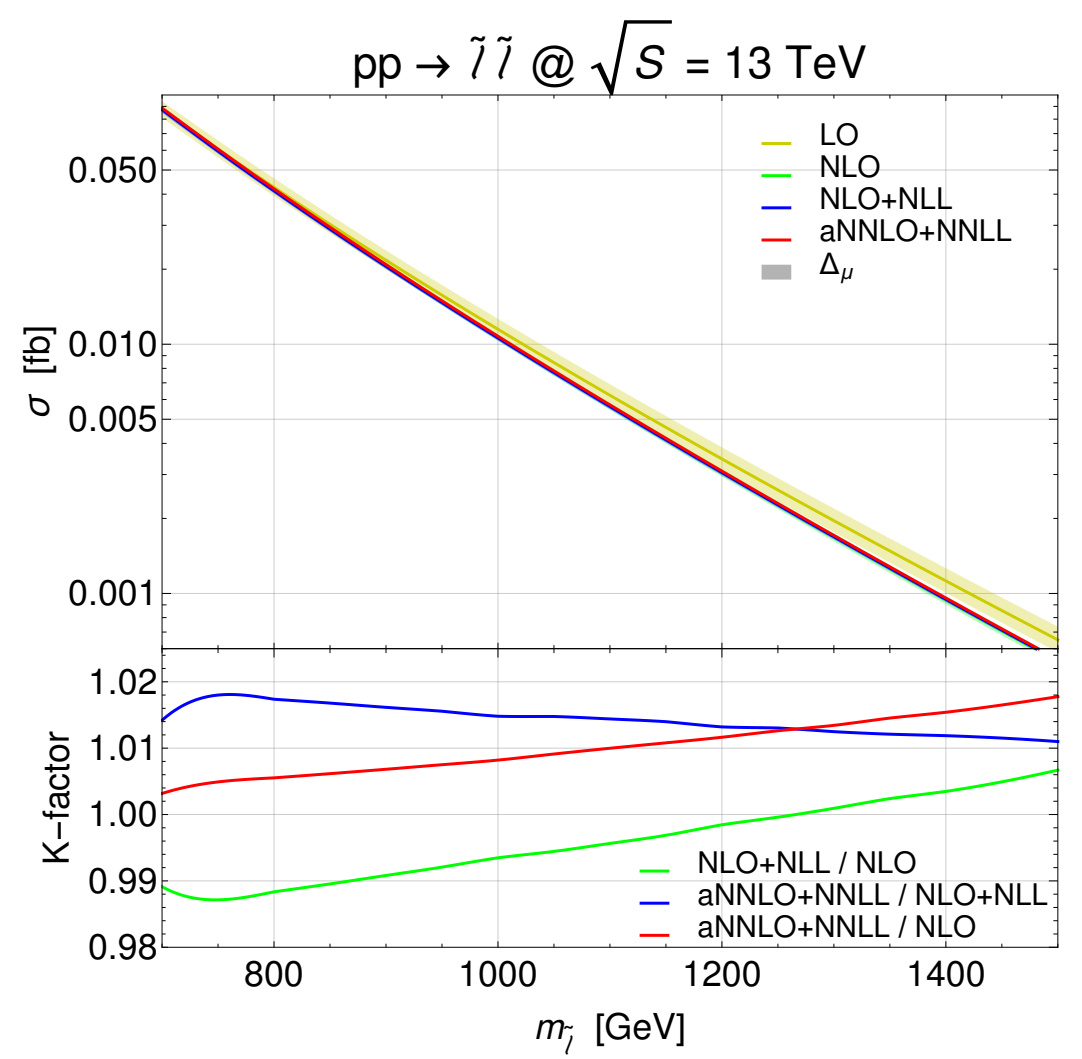

Figure 3. Top: Total cross section for left-handed selectron (or smuon) pair production at the LHC with a center-of-mass energy of $\sqrt{S}=13 \mathrm{TeV}$ as a function of the slepton mass. Shown are results at LO (yellow), NLO (green), NLO+NLL (blue) and aNNLO+NNLL (red) together with the corresponding scale uncertainties (shaded bands). Bottom: Ratios ( $K$ factors) of NLO+NLL over NLO (green), aNNLO+NNLL over NLO+NLL (blue) and aNNLO+NNLL over NLO (red) total cross sections as a function of the slepton mass.

scale (the slepton mass of $1 \mathrm{TeV}$ ). The renormalisation scale is here fixed to this value. While we observe a steeply falling dependence from the PDFs at LO (yellow), it is already partially compensated at NLO through the factorisation of collinear divergences (green), further reduced and somewhat overcompensated at NLO+NLL (blue) and completely flat at aNNLO+NNLL (red).

Figure 4 (bottom) shows the corresponding renormalisation scale dependence, where now the factorisation scale remains fixed. The dependence is only introduced at NLO, where $\alpha_{s}\left(\mu_{R}\right)$ falls with increasing scale (green), since the LO cross section is of electroweak origin (yellow). One then observes an alternating behavior with respect to the sign of the corrections at NLO+NLL (blue) and aNNLO+NNLL (red) with a variation that is reduced from $5 \%$ at NLO to $1 \%$ at aNNLO+NNLL. This demonstrates again the excellent stability of the calculation.

The combined effect of varying the factorisation and renormalisation scales with the seven-point method is shown in figure 5 as a function of the slepton mass in the same range of $700 \mathrm{GeV}$ to $1.5 \mathrm{TeV}$ as considered above. We observe an almost constant theoretical uncertainty of $-3 \%$ to $+1 \%$ at NLO+NLL (blue), which is reduced to about $-0.2 \%$ to 

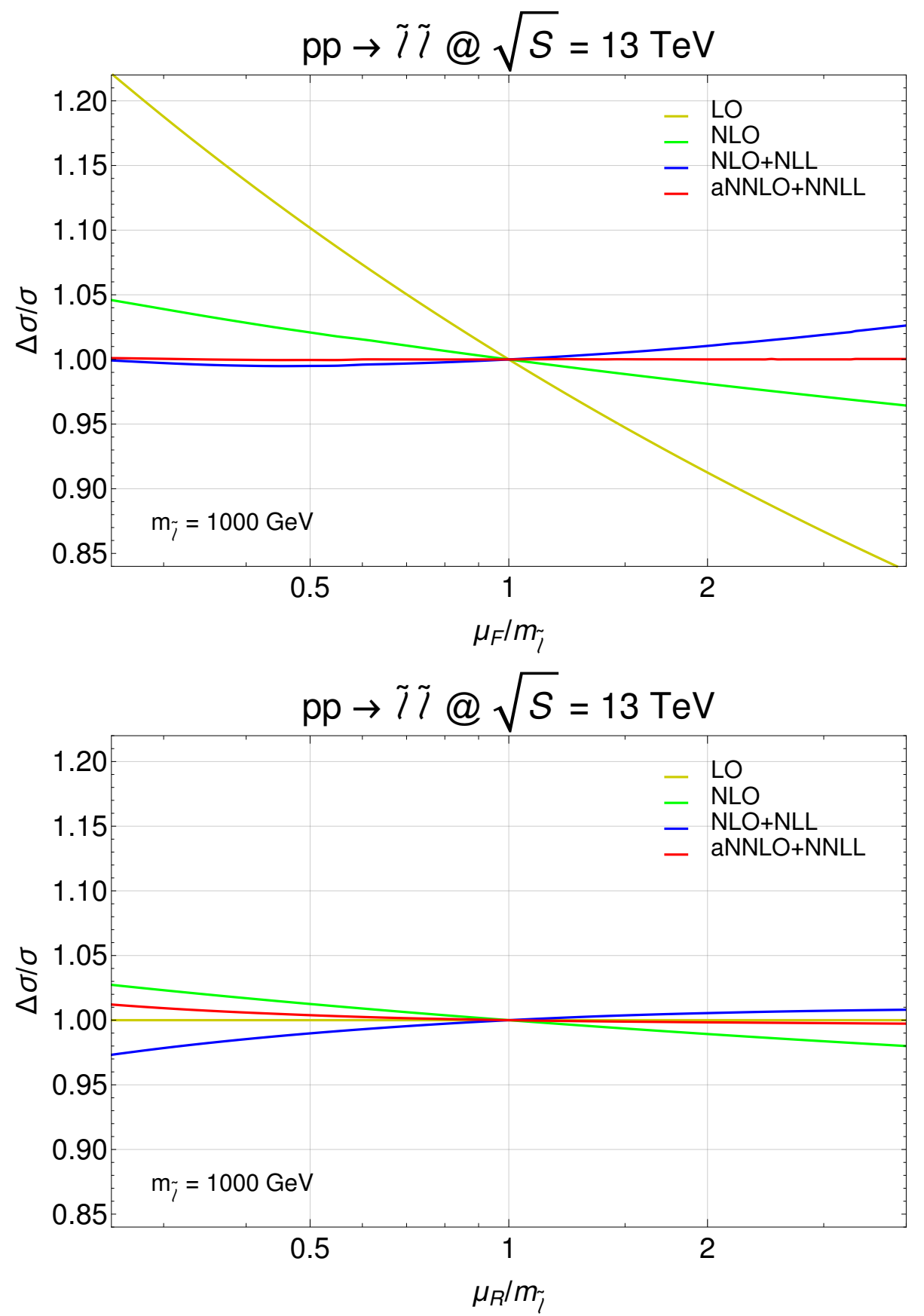

Figure 4. Relative variation of the total cross section for slepton pair production as function of the factorisation (top) and renormalisation scale (bottom). Shown are results at LO (yellow), NLO (green), NLO+NLL (blue) and aNNLO+NNLL (red). 


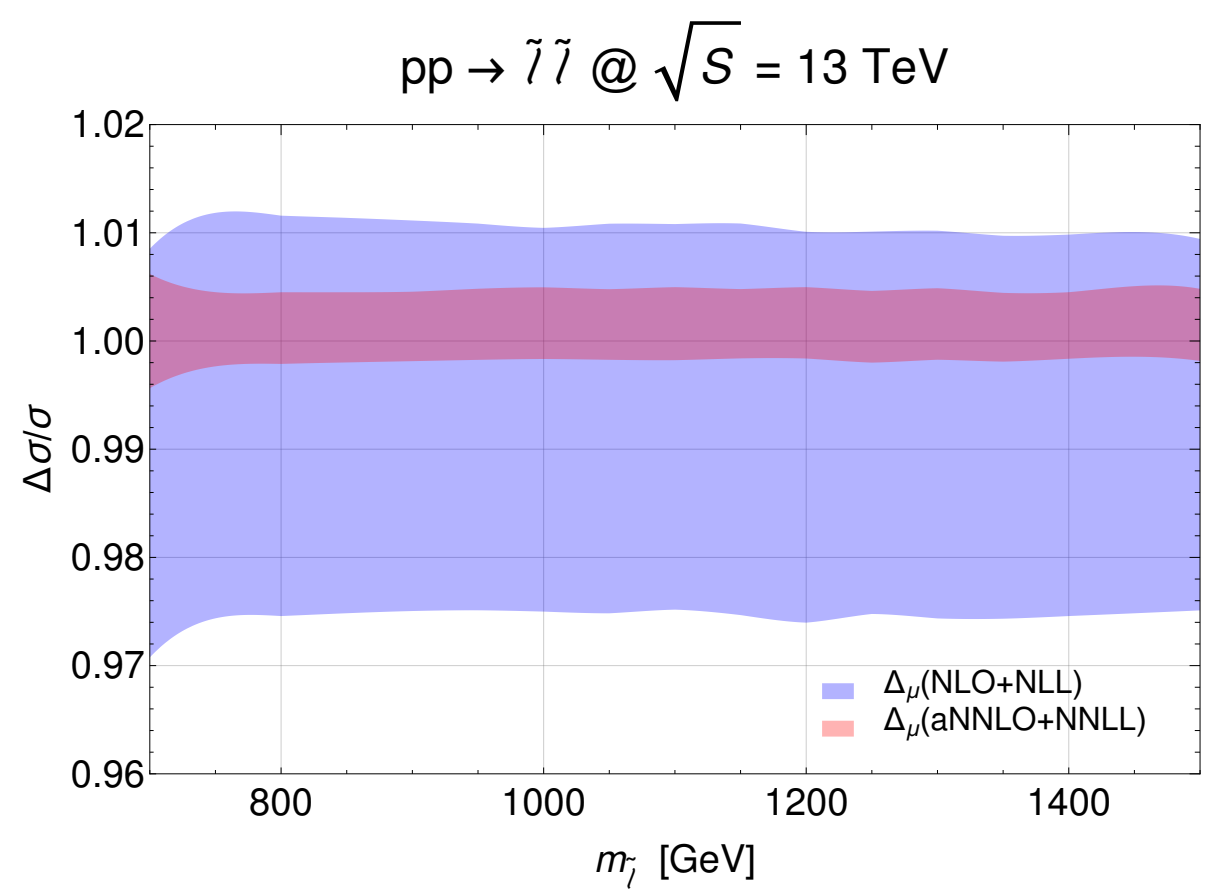

Figure 5. Scale uncertainty of the total cross section for left-handed selectron (or smuon) pair production at the LHC with a center-of-mass energy of $\sqrt{S}=13 \mathrm{TeV}$ as a function of the slepton mass. Shown are the results at NLO+NLL (blue) and aNNLO+NNLL (red shaded band).

$+0.4 \%$ at aNNLO+NNLL (red shaded band) and which is only slightly larger for small slepton masses.

The virtual corrections at NLO do not only introduce a dependence on the renormalisation scale, but - through the squarks and gluinos appearing in the loops - also a weak dependence on other SUSY masses. Resumming logarithmically enhanced or adding approximate NNLO QCD, but not NNLO SUSY-QCD contributions does not alter this dependence significantly. The squark mass dependence of the NLO SUSY-QCD corrections has been studied explicitly in the upper part of figure 4 of ref. [13] and verified by us in figure 7 of ref. [15]. A new direct comparison with ref. [13] within the context of this work found full agreement in the shape. The overall normalisation came out slightly differently since CTEQ4L/M PDFs as used in ref. [13] are not available in LHAPDF as now implemented in RESUMMINO and we therefore had to use newer PDFs (see section 3.1). In figure 6 we show the impact of other SUSY masses on the slepton pair production cross section at the LHC i.e. for both squark and gluino masses and also for much larger values, as a colour-coded ratio of NLO over LO cross sections in the squark-gluino mass plane. Overall, the dependence is weak, as the $K$ factor varies only from 1.170 to 1.186, i.e. by less than two percent. When the squark mass crosses the slepton mass at $1 \mathrm{TeV}$, the threshold behaviour in the triangle loop is clearly visible and represents the dominant dependence. The gluino mass appears only between the incoming (s)quarks in the vertex correction and is clearly less important. The squarks and gluinos decouple and no longer influence the cross section, when their masses reach the multi-TeV scale. 


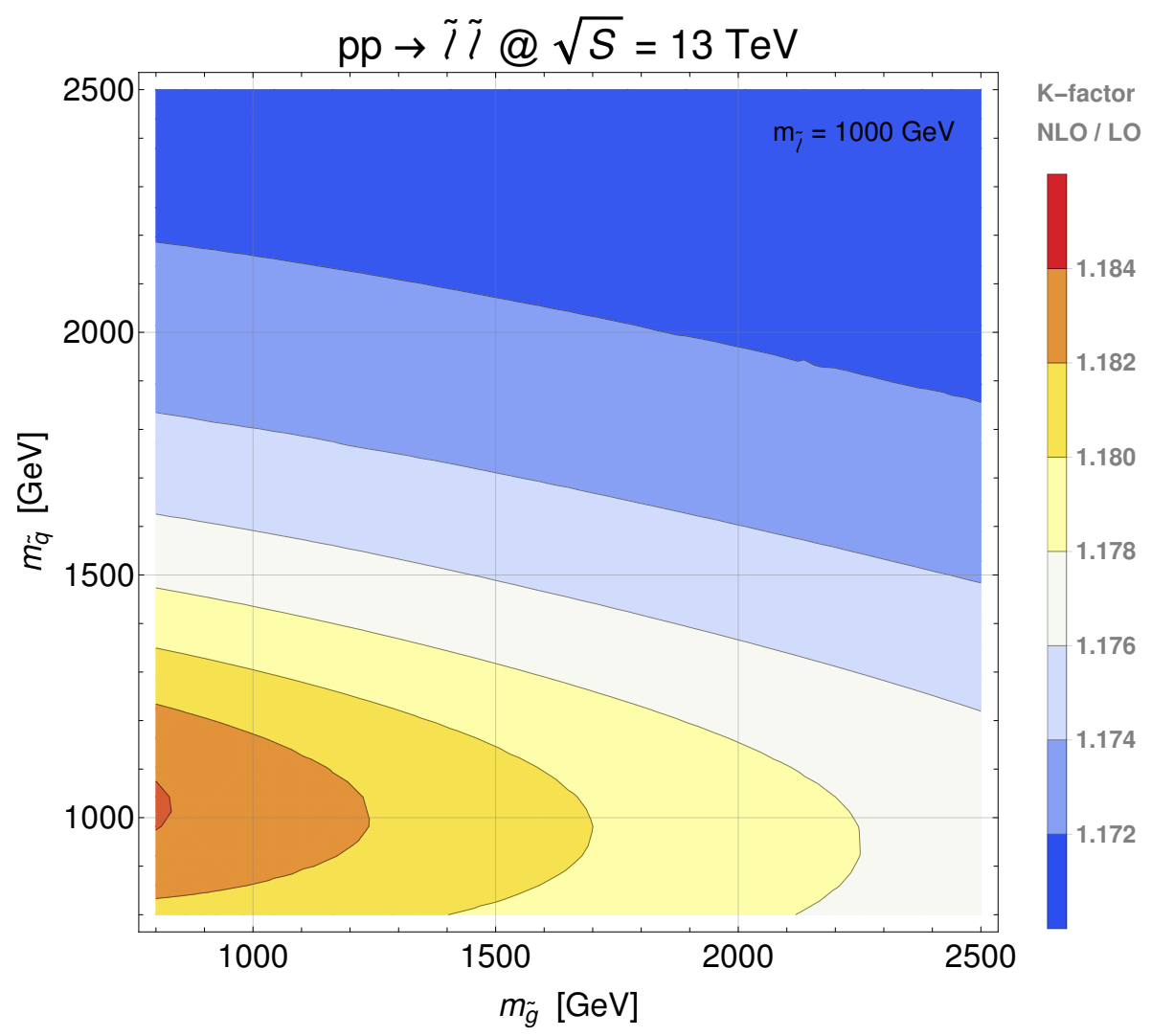

Figure 6. Ratio ( $K$ factor) of NLO over LO total cross sections (both with NLO PDFs) for lefthanded selectron (or smuon) pair production at the LHC with a center-of-mass energy of $\sqrt{S}=$ $13 \mathrm{TeV}$ as a function of the squark and gluino masses.

In simplified scenarios such as the phenomenological Minimal Supersymmetric Standard Model (pMSSM) [74, 75], it is common to assume a degeneracy of sfermion masses. For the first two generations, it is then a good approximation to do so also for the superpartners of the left- and right-handed fermions, since the off-diagonal terms in the sfermion mass matrix are proportional to the corresponding fermion mass. This is different for the third generation, where in the off-diagonal entries of the squark mass matrix the heavyquark masses $m_{t}$ or $m_{b}$ multiply the combinations

$$
m_{L R}=A_{0}-\mu^{*} \begin{cases}\cot \beta & \text { for up }- \text { type sfermions } \\ \tan \beta & \text { for down }- \text { type sfermions }\end{cases}
$$

of the trilinear coupling $A_{0}$, the higgsino mass parameter $\mu$ and the ratio of Higgs vacuum expectation values $\tan \beta=v_{u} / v_{d}$ for stops and sbottoms, respectively. While stops do not enter our calculations, sbottom mixing can influence slepton pair production at NLO. This is demonstrated in figure 7 , where we show the dependence of the total slepton production cross section on the trilinear coupling $A_{0}$ normalised to the cross section at the default value of $-500 \mathrm{GeV}$. The other relevant SUSY parameters have been set to $m_{L L} \simeq m_{R R} \simeq 1.1 \mathrm{TeV}, \mu \simeq 0.8 \mathrm{TeV}$ and $\tan \beta=40$. As expected, one observes an even 


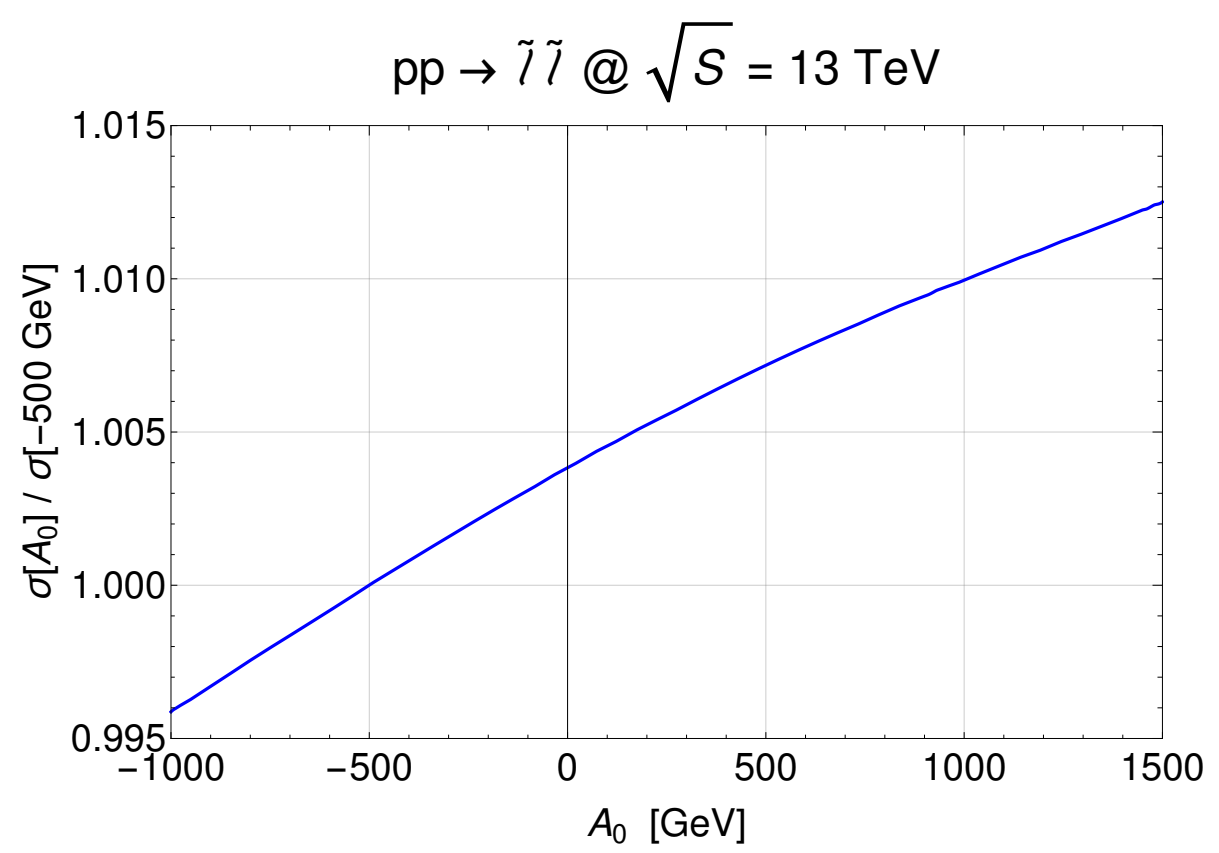

Figure 7. Dependence of the NLO (or NLO+NLL or aNNLO+NNLL) total cross section on the common trilinear coupling $A_{0}$ that governs squark mixing in the sbottom sector. Shown is the ratio over the default scenario with $A_{0}=-500 \mathrm{GeV}$.

weaker dependence of the NLO cross section on the sbottom mixing than on the squark and gluino masses, as it varies only from -0.4 to +1.2 percent.

\section{Conclusion}

In conclusion, we have presented in this paper a calculation of threshold resummation effects on slepton pair production at the LHC with NNLL accuracy matched to approximate NNLO QCD corrections. We collected the relevant analytical results from the literature and described the procedures, with which we matched resummation and fixed-order results and performed the transformation of PDFs and hadronic cross sections to and from Mellin space. Numerically, we found only very moderate increases of invariant-mass distributions and total cross sections with respect to our previous calculations with NLO+NLL precision. More importantly, we observed very significant reductions on the renormalisation and factorisation scale dependences, that now stabilise our predictions to the permil level. We also discussed briefly the dependence of the cross section on squark and gluino masses that enter through virtual loop diagrams at NLO and demonstrated that our calculations are also applicable to mixing squarks, in particular of the third generation. Our results have been implemented in the code RESUMMINO and will soon become available with the next public release. 


\section{Acknowledgments}

We thank B. Fuks for his collaboration on the off-diagonal squark loop contributions and N. Kidonakis for useful discussions. This work has been supported by the BMBF under contract 05H18PMCC1 and the DFG through the Research Training Network 2149 "Strong and weak interactions - from hadrons to dark matter".

Open Access. This article is distributed under the terms of the Creative Commons Attribution License (CC-BY 4.0), which permits any use, distribution and reproduction in any medium, provided the original author(s) and source are credited.

\section{References}

[1] H.P. Nilles, Supersymmetry, Supergravity and Particle Physics, Phys. Rept. 110 (1984) 1 [INSPIRE].

[2] H.E. Haber and G.L. Kane, The Search for Supersymmetry: Probing Physics Beyond the Standard Model, Phys. Rept. 117 (1985) 75 [InSPIRE].

[3] J.A. Aguilar-Saavedra et al., Supersymmetry parameter analysis: SPA convention and project, Eur. Phys. J. C 46 (2006) 43 [hep-ph/0511344] [InSPIRE].

[4] ATLAS collaboration, Search for squarks and gluinos in final states with jets and missing transverse momentum using $139 \mathrm{fb}^{-1}$ of $\sqrt{s}=13 \mathrm{TeV}$ pp collision data with the ATLAS detector, ATLAS-CONF-2019-040 (2019).

[5] CMS collaboration, Search for supersymmetry in proton-proton collisions at $13 \mathrm{TeV}$ in final states with jets and missing transverse momentum, JHEP 10 (2019) 244

[arXiv: 1908.04722] [INSPIRE].

[6] ATLAS collaboration, Search for electroweak production of charginos and sleptons decaying into final states with two leptons and missing transverse momentum in $\sqrt{s}=13 \mathrm{TeV} \mathrm{pp}$ collisions using the ATLAS detector, Eur. Phys. J. C 80 (2020) 123 [arXiv:1908.08215] [INSPIRE].

[7] CMS collaboration, Search for supersymmetric partners of electrons and muons in proton-proton collisions at $\sqrt{s}=13$ TeV, Phys. Lett. B 790 (2019) 140 [arXiv:1806.05264] [INSPIRE].

[8] ATLAS collaboration, Search for direct stau production in events with two hadronic tau leptons in $\sqrt{s}=13 \mathrm{TeV}$ pp collisions with the ATLAS detector, ATLAS-CONF-2019-018 (2019).

[9] CMS collaboration, Search for supersymmetry with a compressed mass spectrum in events with a soft $\tau$ lepton, a highly energetic jet and large missing transverse momentum in proton-proton collisions at $\sqrt{s}=13$ TeV, Phys. Rev. Lett. 124 (2020) 041803 [arXiv: 1910.01185] [INSPIRE].

[10] S. Dawson, E. Eichten and C. Quigg, Search for Supersymmetric Particles in Hadron Hadron Collisions, Phys. Rev. D 31 (1985) 1581 [INSPIRE].

[11] G. Bozzi, B. Fuks and M. Klasen, Slepton production in polarized hadron collisions, Phys. Lett. B 609 (2005) 339 [hep-ph/0411318] [INSPIRE]. 
[12] H. Baer, B.W. Harris and M.H. Reno, Next-to-leading order slepton pair production at hadron colliders, Phys. Rev. D 57 (1998) 5871 [hep-ph/9712315] [INSPIRE].

[13] W. Beenakker, M. Klasen, M. Krämer, T. Plehn, M. Spira and P.M. Zerwas, The production of charginos/neutralinos and sleptons at hadron colliders, Phys. Rev. Lett. 83 (1999) 3780 [Erratum ibid. 100 (2008) 029901] [hep-ph/9906298] [INSPIRE].

[14] G. Bozzi, B. Fuks and M. Klasen, Transverse-momentum resummation for slepton-pair production at the CERN LHC, Phys. Rev. D 74 (2006) 015001 [hep-ph/0603074] [INSPIRE].

[15] G. Bozzi, B. Fuks and M. Klasen, Threshold Resummation for Slepton-Pair Production at Hadron Colliders, Nucl. Phys. B 777 (2007) 157 [hep-ph/0701202] [InSPIRE].

[16] B. Fuks, M. Klasen, D.R. Lamprea and M. Rothering, Revisiting slepton pair production at the Large Hadron Collider, JHEP 01 (2014) 168 [arXiv: 1310.2621] [INSPIRE].

[17] G. Bozzi, B. Fuks and M. Klasen, Joint resummation for slepton pair production at hadron colliders, Nucl. Phys. B 794 (2008) 46 [arXiv:0709.3057] [InSPIRE].

[18] M. Bonvini et al., Parton distributions with threshold resummation, JHEP 09 (2015) 191 [arXiv: 1507.01006] [INSPIRE].

[19] J. Fiaschi and M. Klasen, Slepton pair production at the LHC in NLO+NLL with resummation-improved parton densities, JHEP 03 (2018) 094 [arXiv: 1801.10357] [INSPIRE].

[20] J. Fiaschi and M. Klasen, Electroweakino and slepton pair production at the LHC in $N L O+N L L$ with resummation-improved PDFs, in 2019 European Physical Society Conference on High Energy Physics (EPS-HEP2019) Ghent, Belgium, July 10-17, 2019, arXiv: 1909.05652 [INSPIRE].

[21] J. Debove, B. Fuks and M. Klasen, Transverse-momentum resummation for gaugino-pair production at hadron colliders, Phys. Lett. B 688 (2010) 208 [arXiv:0907.1105] [INSPIRE].

[22] J. Debove, B. Fuks and M. Klasen, Threshold resummation for gaugino pair production at hadron colliders, Nucl. Phys. B 842 (2011) 51 [arXiv: 1005.2909] [INSPIRE].

[23] J. Debove, B. Fuks and M. Klasen, Joint Resummation for Gaugino Pair Production at Hadron Colliders, Nucl. Phys. B 849 (2011) 64 [arXiv: 1102.4422] [INSPIRE].

[24] B. Fuks, M. Klasen, D.R. Lamprea and M. Rothering, Gaugino production in proton-proton collisions at a center-of-mass energy of 8 TeV, JHEP 10 (2012) 081 [arXiv:1207.2159] [INSPIRE].

[25] B. Fuks, M. Klasen, D.R. Lamprea and M. Rothering, Precision predictions for direct gaugino and slepton production at the LHC, Nucl. Part. Phys. Proc. 273-275 (2016) 479 [arXiv: 1407.7963] [INSPIRE].

[26] J. Fiaschi and M. Klasen, Neutralino-chargino pair production at NLO+NLL with resummation-improved parton density functions for LHC Run II, Phys. Rev. D 98 (2018) 055014 [arXiv: 1805.11322] [INSPIRE].

[27] B. Fuks, M. Klasen and M. Rothering, Soft gluon resummation for associated gluino-gaugino production at the LHC, JHEP 07 (2016) 053 [arXiv: 1604.01023] [INSPIRE].

[28] M. Klasen, B. Fuks and M. Sunder, Precision predictions for associated gluino-gaugino production at the LHC, PoS(EPS-HEP2017) 298 [arXiv:1709.02680] [INSPIRE].

[29] B. Fuks, M. Klasen, S. Schmiemann and M. Sunder, Realistic simplified gaugino-higgsino models in the MSSM, Eur. Phys. J. C 78 (2018) 209 [arXiv:1710.09941] [InSPIRE]. 
[30] B. Fuks, M. Klasen, F. Ledroit, Q. Li and J. Morel, Precision predictions for $Z^{\prime}$-production at the CERN LHC: QCD matrix elements, parton showers and joint resummation, Nucl. Phys. B 797 (2008) 322 [arXiv:0711.0749] [INSPIRE].

[31] T. Jezo, M. Klasen, D.R. Lamprea, F. Lyonnet and I. Schienbein, $N L O+N L L$ limits on $W^{\prime}$ and $Z^{\prime}$ gauge boson masses in general extensions of the Standard Model, JHEP 12 (2014) 092 [arXiv: 1410.4692] [INSPIRE].

[32] T. Jezo, M. Klasen, D. Lamprea, F. Lyonnet and I. Schienbein, $N L O+N L L$ limits on $W^{\prime}$ and Z' gauge boson masses in general extensions of the Standard Model, PoS(DIS2015) 112.

[33] M. Klasen, F. Lyonnet and F.S. Queiroz, $N L O+N L L$ collider bounds, Dirac fermion and scalar dark matter in the B-L model, Eur. Phys. J. C 77 (2017) 348 [arXiv:1607.06468] [INSPIRE].

[34] B. Fuks, M. Klasen, D.R. Lamprea and M. Rothering, Precision predictions for electroweak superpartner production at hadron colliders with Resummino, Eur. Phys. J. C 73 (2013) 2480 [arXiv: 1304.0790] [INSPIRE].

[35] ATLAS collaboration, Search for direct production of electroweakinos in final states with one lepton, missing transverse momentum and a Higgs boson decaying into two b-jets in (pp) collisions at $\sqrt{s}=13 \mathrm{TeV}$ with the ATLAS detector, arXiv:1909.09226 [INSPIRE].

[36] CMS collaboration, Search for new physics in events with two soft oppositely charged leptons and missing transverse momentum in proton-proton collisions at $\sqrt{s}=13 \mathrm{TeV}$, Phys. Lett. B 782 (2018) 440 [arXiv:1801.01846] [INSPIRE].

[37] X. Cid Vidal et al., Report from Working Group 3, CERN Yellow Rep. Monogr. 7 (2019) 585 [arXiv: 1812.07831] [INSPIRE].

[38] A. Vogt, Next-to-next-to-leading logarithmic threshold resummation for deep inelastic scattering and the Drell-Yan process, Phys. Lett. B 497 (2001) 228 [hep-ph/0010146] [INSPIRE].

[39] N. Kidonakis, A unified approach to NNLO soft and virtual corrections in electroweak, Higgs, QCD and SUSY processes, Int. J. Mod. Phys. A 19 (2004) 1793 [hep-ph/0303186] [INSPIRE].

[40] N. Kidonakis, Collinear and soft gluon corrections to Higgs production at NNNLO, Phys. Rev. D 77 (2008) 053008 [arXiv:0711.0142] [INSPIRE].

[41] W. Beenakker, R. Hopker, M. Spira and P.M. Zerwas, Squark and gluino production at hadron colliders, Nucl. Phys. B 492 (1997) 51 [hep-ph/9610490] [INSPIRE].

[42] W. Beenakker, M. Krämer, T. Plehn, M. Spira and P.M. Zerwas, Stop production at hadron colliders, Nucl. Phys. B 515 (1998) 3 [hep-ph/9710451] [INSPIRE].

[43] W. Beenakker et al., NNLL resummation for squark and gluino production at the LHC, JHEP 12 (2014) 023 [arXiv: 1404.3134] [INSPIRE].

[44] W. Beenakker, C. Borschensky, R. Heger, M. Krämer, A. Kulesza and E. Laenen, NNLL resummation for stop pair-production at the LHC, JHEP 05 (2016) 153 [arXiv:1601.02954] [INSPIRE].

[45] W. Beenakker, C. Borschensky, M. Krämer, A. Kulesza and E. Laenen, NNLL-fast: predictions for coloured supersymmetric particle production at the LHC with threshold and Coulomb resummation, JHEP 12 (2016) 133 [arXiv: 1607.07741] [INSPIRE]. 
[46] A. Broggio, M. Neubert and L. Vernazza, Soft-gluon resummation for slepton-pair production at hadron colliders, JHEP 05 (2012) 151 [arXiv:1111.6624] [INSPIRE].

[47] M. Beneke, P. Falgari and C. Schwinn, Threshold resummation for pair production of coloured heavy (s)particles at hadron colliders, Nucl. Phys. B 842 (2011) 414 [arXiv: 1007.5414] [INSPIRE].

[48] M. Beneke, J. Piclum, C. Schwinn and C. Wever, NNLL soft and Coulomb resummation for squark and gluino production at the LHC, JHEP 10 (2016) 054 [arXiv:1607.07574] [INSPIRE].

[49] A. Broggio, A. Ferroglia, M. Neubert, L. Vernazza and L.L. Yang, Approximate NNLO Predictions for the Stop-Pair Production Cross Section at the LHC, JHEP 07 (2013) 042 [arXiv: 1304.2411] [INSPIRE].

[50] A. Broggio, A. Ferroglia, M. Neubert, L. Vernazza and L.L. Yang, NNLL Momentum-Space Resummation for Stop-Pair Production at the LHC, JHEP 03 (2014) 066 [arXiv: 1312.4540] [INSPIRE].

[51] G.F. Sterman, Summation of Large Corrections to Short Distance Hadronic Cross-Sections, Nucl. Phys. B 281 (1987) 310 [inSPIRE].

[52] S. Catani and L. Trentadue, Resummation of the QCD Perturbative Series for Hard Processes, Nucl. Phys. B 327 (1989) 323 [InSPIRE].

[53] O.V. Tarasov, A.A. Vladimirov and A.Yu. Zharkov, The Gell-Mann-Low Function of QCD in the Three Loop Approximation, Phys. Lett. 93B (1980) 429 [INSPIRE].

[54] S.A. Larin and J.A.M. Vermaseren, The three loop QCD $\beta$-function and anomalous dimensions, Phys. Lett. B 303 (1993) 334 [hep-ph/9302208] [INSPIRE].

[55] S. Moch, J.A.M. Vermaseren and A. Vogt, Higher-order corrections in threshold resummation, Nucl. Phys. B 726 (2005) 317 [hep-ph/0506288] [INSPIRE].

[56] M. Krämer, E. Laenen and M. Spira, Soft gluon radiation in Higgs boson production at the LHC, Nucl. Phys. B 511 (1998) 523 [hep-ph/9611272] [INSPIRE].

[57] S. Catani, D. de Florian and M. Grazzini, Higgs production in hadron collisions: Soft and virtual QCD corrections at NNLO, JHEP 05 (2001) 025 [hep-ph/0102227] [INSPIRE].

[58] S. Catani, D. de Florian, M. Grazzini and P. Nason, Soft gluon resummation for Higgs boson production at hadron colliders, JHEP 07 (2003) 028 [hep-ph/0306211] [INSPIRE].

[59] M. Beneke et al., Leading-logarithmic threshold resummation of the Drell-Yan process at next-to-leading power, JHEP 03 (2019) 043 [arXiv: 1809.10631] [INSPIRE].

[60] M. Beneke, A. Broggio, S. Jaskiewicz and L. Vernazza, Threshold factorization of the Drell-Yan process at next-to-leading power, arXiv:1912.01585 [INSPIRE].

[61] M. Beneke, M. Garny, S. Jaskiewicz, R. Szafron, L. Vernazza and J. Wang, Leading-logarithmic threshold resummation of Higgs production in gluon fusion at next-to-leading power, JHEP 01 (2020) 094 [arXiv:1910.12685] [INSPIRE].

[62] N. Bahjat-Abbas et al., Diagrammatic resummation of leading-logarithmic threshold effects at next-to-leading power, JHEP 11 (2019) 002 [arXiv:1905.13710] [INSPIRE].

[63] T.O. Eynck, E. Laenen and L. Magnea, Exponentiation of the Drell-Yan cross-section near partonic threshold in the DIS and MS-bar schemes, JHEP 06 (2003) 057 [hep-ph/0305179] [INSPIRE]. 
[64] C. Duhr, F. Dulat and B. Mistlberger, The Drell-Yan cross section to third order in the strong coupling constant, arXiv:2001.07717 [INSPIRE].

[65] A.A. H., G. Das, M.C. Kumar, P. Mukherjee, V. Ravindran and K. Samanta, Resummed Drell-Yan cross-section at $N^{3} L L$, arXiv:2001.11377 [INSPIRE].

[66] H. Contopanagos and G.F. Sterman, Principal value resummation, Nucl. Phys. B 419 (1994) 77 [hep-ph/9310313] [INSPIRE].

[67] S. Catani, M.L. Mangano, P. Nason and L. Trentadue, The resummation of soft gluons in hadronic collisions, Nucl. Phys. B 478 (1996) 273 [hep-ph/9604351] [INSPIRE].

[68] A.D. Martin, W.J. Stirling, R.S. Thorne and G. Watt, Parton distributions for the LHC, Eur. Phys. J. C 63 (2009) 189 [arXiv:0901.0002] [INSPIRE].

[69] S. Dulat et al., New parton distribution functions from a global analysis of quantum chromodynamics, Phys. Rev. D 93 (2016) 033006 [arXiv: 1506.07443] [InSPIRE].

[70] J. Harz, B. Herrmann, M. Klasen, K. Kovařík and P. Steppeler, Theoretical uncertainty of the supersymmetric dark matter relic density from scheme and scale variations, Phys. Rev. D 93 (2016) 114023 [arXiv: 1602.08103] [INSPIRE].

[71] J. Branahl, J. Harz, B. Herrmann, M. Klasen, K. Kovař́k and S. Schmiemann, SUSY-QCD corrected and Sommerfeld enhanced stau annihilation into heavy quarks with scheme and scale uncertainties, Phys. Rev. D 100 (2019) 115003 [arXiv:1909.09527] [InSPIRE].

[72] M. Klasen and G. Kramer, Large transverse momentum jet production and DIS distributions of the proton, Phys. Lett. B 386 (1996) 384 [hep-ph/9605210] [INSPIRE].

[73] M. Klasen and G. Kramer, Factorization scheme and scale dependence in diffractive dijet production at low $Q^{2}$, J. Phys. G 31 (2005) 1391 [hep-ph/0506121] [INSPIRE].

[74] MSSM Working Group collaboration, The minimal supersymmetric standard model: Group summary report, in GDR (Groupement De Recherche) - Supersymetrie, Montpellier, France, April 15-17, 1998, 1998, hep-ph/9901246 [INSPIRE].

[75] A. Djouadi, J.-L. Kneur and G. Moultaka, SuSpect: A Fortran code for the supersymmetric and Higgs particle spectrum in the MSSM, Comput. Phys. Commun. 176 (2007) 426 [hep-ph/0211331] [INSPIRE]. 